\title{
Impact of chromosome 17 centromere copy number increase on patient survival and human epidermal growth factor receptor 2 expression in gastric adenocarcinoma
}

\author{
MACIEJ CIESIELSKI ${ }^{1,2}$, MARIUSZ SZAJEWSKI ${ }^{1,2}$, JAKUB WALCZAK ${ }^{1}$, \\ RAFAŁ PECKSA ${ }^{3}$, RADOSŁAW LENCKOWSKI ${ }^{4}$, MAŁGORZATA SUPEŁ ${ }^{4}$, \\ JACEK ZIELIŃSKI $^{5}$ and WIESŁAW JANUSZ KRUSZEWSKI ${ }^{1,2}$ \\ ${ }^{1}$ Department of Oncological Surgery, Gdynia Centre of Oncology, Pomeranian Hospitals, Gdynia, \\ Pomeranian Voivodship 81-519; ${ }^{2}$ Division of Propedeutics of Oncology and ${ }^{3}$ Department of Pathomorphology, \\ Medical University of Gdańsk, Gdańsk, Pomeranian Voivodship 80-210; ${ }^{4}$ Department of Pathomorphology, \\ Gdynia Centre of Oncology, Pomeranian Hospitals, Gdynia, Pomeranian Voivodship 81-519; \\ ${ }^{5}$ Department of Oncological Surgery, Medical University of Gdańsk, Gdańsk, Pomeranian Voivodship 80-210, Poland
}

Received May 19, 2020; Accepted November 16, 2020

DOI: $10.3892 / 01.2020 .12403$

\begin{abstract}
The accurate evaluation of human epidermal growth factor receptor 2 (HER2) status is essential for the appropriate use of targeted therapies. An increased number of chromosome 17 centromere enumeration probe (CEP17) signals may underrate fluorescence in situ hybridization (FISH) outcomes, resulting in false-negative or a false-equivocal HER2 status assessment. The aim of the present study was to assess the frequency of CEP17 copy number increase (CNI), its effects on HER2 protein expression (and the subsequent effects on tumor cells), and the survival outcomes of patients with gastric cancer. Archival primary tumor samples from 244 patients that underwent gastric resection for adenocarcinoma were retrieved for both HER2 protein expression analysis (using immunochemistry) and HER2 gene amplification (using FISH). The associations between HER2 status, CEP17 CNI and multiple clinicopathological parameters (including survival outcome), were assessed. The relationship between CEP17 CNI and HER2 protein upregulation was also investigated. CEP17 CNI was detected in $17.2 \%$ of cases, and a strong
\end{abstract}

Correspondence to: Dr Maciej Ciesielski, Department of Oncological Surgery, Gdynia Centre of Oncology, ul. Powstania Styczniowego 1, Pomeranian Hospitals, Gdynia, Pomeranian Voivodship 81-519, Poland

E-mail: maccies@gumed.edu.pl

Abbreviations: HER2, human epidermal growth factor receptor 2; $\mathrm{CNI}$, copy number increase; CEP17, chromosome 17 centromere enumeration probe; IHC, immunochemistry; FISH, fluorescence in situ hybridization; MLPA, multiplex ligation-dependent probe amplification; CAP/ASCP/ASCO, College of American Pathologists, American Society for Clinical Pathology and the American Society of Clinical Oncology

Key words: stomach neoplasm, genes, erbB-2, human chromosome pair 17, fluorescence in situ hybridization association between CEP17 CNI and HER2 upregulation was revealed. The impact of CEP17 CNI on survival did not reach statistical significance. Consequently, CEP17 CNI was discovered to be strongly associated with HER2 upregulation in tumor cells, which may characterize a critical issue in HER2 testing. Therefore, the eligibility for HER2-targeted agents in CEP17 CNI-positive patients warrants further recognition.

\section{Introduction}

Despite declining incidence, gastric cancer remains one of the leading causes of cancer-associated death worldwide, with the proportion of deaths to newly diagnosed cases exceeding $75 \%$ in 2018 (1). The late onset of clinical symptoms limits the curative role of surgical treatment and standard chemotherapy. However, there is evidence of improvements in patient survival resulting from the implementation of targeted treatment. Among numerous monoclonal antibodies applied in the treatment of gastric cancer to date, trastuzumab is still the only standard agent that demonstrates significant efficacy (2). Trastuzumab is a recombinant humanized monoclonal antibody targeting human epidermal growth factor receptor 2 (HER2) that has been demonstrated to improve survival outcome in patients with HER2-positive gastric cancer $(2,3)$.

$H E R 2$, also known as ERBB2 and HER2/neu, is a proto-oncogene located on the long arm of chromosome 17. HER 2 encodes the tyrosine kinase membrane receptor HER2, whose phosphorylation initiates signaling pathways resulting in cell division, proliferation, differentiation and the suppression of apoptosis (4-7). HER2 is expressed in a variety of tissues, including the breast and gastrointestinal tract, where it is considered one of the key drivers of tumorigenesis (3-5). The upregulation of HER2, or amplification of the HER2 gene, is associated with significantly worse prognosis in patients with breast cancer, both via enhanced local growth and metastasis formation (5). In gastric cancer, studies of the prognostic relevance of HER2 upregulation/amplification have generated 
inconsistent results, and the association between HER2 status and gastric cancer prognosis remain controversial $(4,5)$.

Trastuzumab is used to target the extracellular domain of HER2, which inhibits HER2-mediated downstream signal activation $(4,5)$. Trastuzumab was originally introduced to treat HER2-positive metastatic breast cancer $(5,6)$. In the case of patients with advanced HER2-positive gastric adenocarcinoma, it has also been acknowledged that the addition of trastuzumab to the chemotherapy regimen increases the response rate and prolongs both progression-free and overall survival time (4).

The accurate evaluation of HER 2 status is essential for the appropriate use of anti-HER2 therapy $(8,9)$. To assess HER2 positivity, protein expression is evaluated by immunohistochemistry (IHC) and if the result is equivocal (2+), fluorescence in situ hybridization (FISH) is performed to assess HER2 gene amplification (4-6). Generally speaking, in situ hybridization is performed using a single probe, in which absolute counts per cell determine the scoring system, or with the use of a dual probe technique that relies on the HER2/chromosome 17 centromere enumeration probe (CEP17) ratio $(4,10)$. In gastric cancer, the dual probe hybridization method is strongly recommended (4); single probe methods are discouraged as they are more affected by section thickness $(4,10)$, tumor mitotic index and abnormal chromosome copy number (10). However, in dual probe methods, the increased number of CEP17 signals (often classified as polysomic) may underrate the test results.

The negative impact of CEP17 copy number increase (CNI) on prognosis has previously been revealed in cases of breast cancer $(11,12)$. Our previous study (13) demonstrated that CEP17 CNI may also be a negative prognostic factor in gastric cancer, but the studied group was relatively small. Thus, the aim of the present study was to assess the frequency of CEP17 CNI occurrence and its effect on HER2 protein expression in tumor cells, as well as treatment outcome, in a larger group of patients with gastric cancer.

\section{Materials and methods}

Study design and participants. Our previous study was performed on 83 patients who underwent surgery between July 2006 and January 2011 at the Department of Surgical Oncology of Gdynia Oncology Centre (Poland) (13). To increase the size of the study group, patients that received surgery in the same center between January 2011 and December 2013, and patients from the Department of Oncological Surgery, Medical University of Gdańsk (operated upon between July 2006 and December 2013), were also included. Both inclusion and exclusion criteria, as well as IHC and FISH methodology, were the same for both the old and new cohorts ( 83 and 208 patients, respectively).

The archival primary tumor samples from the additional 208 patients (who underwent major gastric resection for adenocarcinoma) were retrospectively retrieved for both HER2 protein expression analysis by IHC, and HER2 gene amplification using FISH. The combined study group consisted of 291 patients that underwent major gastric resection for adenocarcinoma of the stomach. The only inclusion criterion for patients was major resection for adenocarcinoma of the stomach in the study period. The exclusion criterion was the coexistence of any other types of malignancy, including stromal tumors, neuroendocrine cancer and lymphoma.

The surgical and pathological reports were analyzed and included the following study parameters: i) Range of stomach resection (total or subtotal); ii) extent of lymphadenectomy; iii) the total number of harvested lymph nodes; iv) pTNM stage of the disease, according to the 7 th edition of the American Society for Clinical Pathology and the American Joint Committee on Cancer Staging manual (14); v) depth of tumor invasion into the stomach wall (pT); vi) presence of nodal involvement $(\mathrm{pN})$; vii) number of metastatic lymph nodes; viii) presence of distant metastases (M); ix) Lauren histological type of tumor; $x$ ) presence of mucinous component in the tumor tissue; xi) tumor location in the stomach (cardia involvement); and xii) survival outcome. Mortality data were acquired from the Polish Ministry of Digitization on January 1st, 2019.

HER2 status was evaluated according to the guidelines from the College of American Pathologists, American Society for Clinical Pathology and the American Society of Clinical Oncology (CAP/ASCP/ASCO) (4). HER2 status was considered positive in cases of IHC results of $3+$, or $2+$ with the presence of HER2 gene amplification (FISH-positive). The associations between HER2 status, CEP17 CNI and multiple clinicopathological parameters (including survival) were assessed, as well as the relationship between CEP17 CNI and HER2 protein upregulation.

For statistical reasons, patients with TNM stage I or II disease were combined into one group, and those with TNM stage III or IV into a second group. Similarly, pT1 and pT2 were combined into one group and pT3 and pT4 into a second. Patients with Lauren type II or III classification were classified as 'diffuse type'.

Preoperative diagnosis and surgery. The patients were preoperatively diagnosed by endoscopy with histopathological examination. The stage of the disease was routinely determined by abdominal CT and chest radiography. The standard surgical procedure for gastric cancer in both centers was total gastrectomy with appropriate lymphadenectomy. The particular extent of gastric resection and lymph node dissection was based on the disease stage and the individual surgeon's judgement. Resection was routinely followed by Roux-en-Y reconstruction. All procedures were performed by laparotomy. Resected tissue was fixed in $10 \%$ neutral buffered formalin at room temperature for at least $24 \mathrm{~h}$.

IHC. IHC staining was conducted on 4- $\mu \mathrm{m}$ tissue sections which were obtained from paraffin-embedded tissue blocks. The sample collection took place between January and March 2018, and the requirement for patient consent was waived by the Ethics Committee of the Medical University of Gdańsk. The sections containing the most representative tumor tissues, without signs of necrosis, were selected and placed in silanized glasses. Then deparaffinization with use of xylene), rehydration with use of descending alcohol series (ethanol 99, 95 and 80\%) and blocking using of 3\% hydrogen peroxide solution for $4 \mathrm{~min}$ at $36^{\circ} \mathrm{C}$ were performed, and the glasses were incubated at $36^{\circ} \mathrm{C}$ for $24 \mathrm{~h}$. For HER2 staining, pre-diluted anti-HER2/neu (4B5) Rabbit Monoclonal Primary 
Antibody (cat. no. 05278368001; Roche Diagnostics) was used in an automatic machine (Roche Benchmark GX; Roche Diagnostics), according to the manufacturer's instructions. The Benchmark machine performed a fully automated heat induced epitope retrieval step with use of pre-diluted ready to use Cell Conditioning 1 (cat. no. 950-124; Ventana; Roche Diagnostics) at $95^{\circ} \mathrm{C}$ for $35 \mathrm{~min}$. The antigen visualization was performed via the iVIEW DAB detection kit (streptavidin-horseradish peroxidase conjugate; cat. no. 760-091; Ventana; Roche Diagnostics) at $37^{\circ} \mathrm{C}$ for $32 \mathrm{~min}$. The samples were counterstained with hematoxylin II (cat. no. 760-2021; Ventana; Roche Diagnostics) at room temperature for $4 \mathrm{~min}$, blued with Bluing Reagent (cat. no. 760-2037; Ventana; Roche Diagnostics) at room temperature for $4 \mathrm{~min}$ in a fully automated way. The tissue sections were then dehydrated in an ascending alcohol series $(80,95,99$ and $99 \%)$ and xylene and placed on coverslips. For evaluation, the Olympus BX43 light microscope (magnification, $\mathrm{x} 40$; Olympus Corporation) was used according to the criteria recommended by Hofmann et al (15), and the evaluation was confirmed by Abrahao-Machado (Department of Pathology, Barretos Cancer Hospital, Barretos, Brazil) and Rushoff (Targos Molecular Pathology GmBH und Pathology Nordhessen, Kassel, Germany) (6,7).

FISH. Molecular cytogenetic analysis was performed in all cases (irrespective of IHC score) at the Molecular Oncology and Genetics Department, IFM, Łukaszczyk Oncology Centre in Bydgoszcz; 4- and 6- $\mu \mathrm{m}$ sections from formalin-fixed paraffin-embedded tissue blocks were used for FISH analysis. The most representative areas of the tumor (without signs of necrosis) were selected, and HER2 gene amplification was performed. The commercially validated Vysis PathVysion HER2 FISH test (Vysis, Inc.; Abbott Pharmaceutical) was used to evaluate gene amplification per the manufacturer's protocol.

The tissue sections were deparaffinized, dehydrated and air-dried. After immersion in $0.2 \mathrm{~N} \mathrm{HCl}$, purified water and Wash Buffer, the samples were pretreated with Pretreatment Solution at $80^{\circ} \mathrm{C}$ for $30 \mathrm{~min}$. The sections were then immersed in Protease Solution at $37^{\circ} \mathrm{C}$ for $34 \mathrm{~min}$, followed by immersion in Wash Buffer (70, 80 and 100\% ethanol), and then subjected to hybridization. The DNA probe mixture (10 ng/ $\mu 1226 \mathrm{~kb}$ HER 2 probe and $20 \mathrm{ng} / \mu 19 \mathrm{~kb}$ CEP17 probe) was applied to the target area of the slide and covered with a glass coverslip (both probes were fragmented to facilitate hybridization). After the probe mixture had spread evenly under the coverslip, the slides were placed in a prewarmed humidified hybridization chamber and incubated at $74^{\circ} \mathrm{C}$ for $5 \mathrm{~min}$, and then at $37^{\circ} \mathrm{C}$ overnight. Next, the slides were immersed in post-hybridization wash buffer at room temperature for $15 \mathrm{~min}$, and then in prewarmed post-hybridization buffer at $72^{\circ} \mathrm{C}$ for $2 \mathrm{~min}$. After air-drying, $10 \mu \mathrm{l}$ DAPI was added to the target area and a glass coverslip was applied. The slides were stored in the dark prior to signal enumeration.

A minimum of 60 cells in interphase were scored for each sample using a fluorescence microscope (Eclipse 80i; Nikon Corporation) and CAP/ASCP/ASCO 2016 HER2 standard recommendations (4). A geneticist (Professor Marzena Anna Lewandowska; Molecular Oncology and Genetics Department, IFM, Łukaszczyk Oncology Centre in Bydgoszcz) reported the average copy number of HER2 and CEP17, and the HER2/CEP17 ratio in each case. FISH results were interpreted as positive with a ratio of $H E R 2$ to CEP17 signal $\geq 2$, and negative with a ratio $<2$. In cases with an average of $\geq 3$ CEP17 copies (CEP17 CNI) and a ratio $<2$, the presence of $>6$ HER2 signals was interpreted as a positive result, $<4$ HER 2 signals was interpreted as a negative result, and a signal number between 4 and 6 was interpreted as an equivocal result. Cases with IHC examination results of $2+$ and an equivocal FISH result were considered as undetermined HER2 status, and were not included in the statistical analysis for the relationship between HER2 status and clinicopathological parameters (Table I). The 83 FISH results used in our previous study (13) were reinterpreted according to the same recommendations. Images were captured using Lucia Cytogenetics 2 Laboratory Imaging software v.2.1, examples of which are presented in Fig. 1.

Statistical analysis. Statistical analysis was performed using Statistica software, version 13 (StatSoft, Inc.; Dell). Survival analysis was calculated using the Kaplan-Meier method, followed by the log-rank test, to assess the differences between the groups. The clinicopathological variables of the four patient groups were compared using $\chi^{2}$, Fisher's exact or U Mann-Whitney tests, as appropriate. $\mathrm{P}<0.05$ was considered to indicate a statistically significant difference.

\section{Results}

Data collection. The IHC or FISH assays were unsuccessful in 22 cases. The most common reasons were either inefficient material (in the case of small tumors) or invalid material preservation, most often within the 2006-2008 period, which was similar to the results of our previous study (25 unsuccessful cases). Results were ultimately obtained for 186 patients; the complete and successfully tested group consisted of 58 old and 186 new cases $(n=244)$.

Treatment details. Among the studied group, 213 patients (87.3\%) underwent total gastrectomy and 31 (12.7\%) underwent subtotal gastric resection. The range of lymphadenectomy was D2 in $34(13.9 \%)$, D1+ in $25(10.2 \%)$, D1 in $179(73.4 \%)$ and D0 in $6(2.5 \%)$ cases (16). There were $200(82.0 \%)$ procedures with curative intent and $44(18.0 \%)$ regarded as palliative. The average number of resected lymph nodes was 21.1 (median, 19.5; and range, 0-78), and neoadjuvant chemotherapy was administered to $8.2 \%$ of patients.

CEP17 CNI rate and its association with clinicopathological features. CEP17 CNI was observed in $17.2 \%$ of cases. There were no significant differences in the CEP17-positive and -negative groups concerning the range of stomach resection (rate of total gastrectomy, $90.5 \%$ vs. $86.6 \%$ ), the extent of lymphadenectomy (rate of D2-D1+, 26.2 vs. 23.8\%), the total number of lymph nodes resected (mean, 18.9 vs. 21.5; median, 19.5 vs. 19.5), pTNM stage, the depth of tumor invasion into the stomach wall ( $\mathrm{pT})$, the presence of nodal involvement $(\mathrm{pN})$, the number of metastatic lymph nodes, the presence of distant metastases (M), Lauren histological type of the tumor or the presence of mucinous component in the tumor cells (Table I). 
Table I. Association between HER2 status or CEP17 CNI and clinicopathological parameters.

\begin{tabular}{|c|c|c|c|c|c|c|}
\hline \multirow[b]{2}{*}{ Clinicopathological feature } & \multicolumn{2}{|c|}{ CEP17 CNI (+), CEP17 CNI (-), } & \multicolumn{4}{|c|}{$\operatorname{HER} 2(+), \operatorname{HER} 2(-)}$, \\
\hline & $\mathrm{n}=42$ & $n=202$ & P-value & $\mathrm{n}=28$ & $\mathrm{n}=212$ & P-value \\
\hline Rate of total gastrectomy, $\%$ & 90.5 & 86.6 & 0.49 & 89.3 & 87.3 & 0.89 \\
\hline Rate of D2-D1+ lymphadenectomy, \% & 26.2 & 23.8 & 0.73 & 17.9 & 25.0 & 0.4 \\
\hline Total number of lymph nodes resected mean/median & $18.9 / 21.5$ & $19.5 / 19.5$ & 0.33 & $18.8 / 21.5$ & $16.0 / 20.0$ & 0.17 \\
\hline pT3-pT4, \% & 81.0 & 73.8 & 0.600 & 60.7 & 76.9 & 0.120 \\
\hline $\mathrm{pN}+, \%$ & 76.2 & 67.8 & 0.300 & 60.7 & 70.8 & 0.300 \\
\hline Number of metastatic lymph nodes, mean/median & $5.5 / 3$ & $5.9 / 2$ & 0.700 & $4.9 / 1.5$ & $6.1 / 3$ & 0.310 \\
\hline Mucinous component, $\%$ & 23.8 & 31.7 & 0.300 & 10.7 & 33.0 & $0.010^{\mathrm{a}}$ \\
\hline Lauren diffuse type, $\%$ & 45.2 & 50.0 & 0.600 & 25.0 & 52.8 & 0.005 \\
\hline Cardia involvement, $\%$ & 50.0 & 24.8 & 0.001 & 35.7 & 28.3 & 0.400 \\
\hline Presence of distant metastases, $\%$ & 14.3 & 8.4 & 0.200 & 14.3 & 9.0 & $0.300^{\mathrm{a}}$ \\
\hline pTNM III-IV, \% & 59.5 & 55.0 & 0.600 & 42.9 & 58.0 & 0.130 \\
\hline Overall survival mean/median (months) & $35.7 / 17.5$ & $45.1 / 31.5$ & 0.200 & $49.7 / 32.5$ & $43.0 / 29.5$ & 0.510 \\
\hline 1-year survival, $\%$ & 64.3 & 75.2 & 0.120 & 75.0 & 73.6 & 0.790 \\
\hline 2-year survival, $\%$ & 42.9 & 58.4 & 0.050 & 57.1 & 56.1 & 0.810 \\
\hline 3-year survival, \% & 33.3 & 46.5 & 0.070 & 42.9 & 44.8 & 0.940 \\
\hline 4-year survival, \% & 31.0 & 41.6 & 0.100 & 42.9 & 40.1 & 0.680 \\
\hline 5-year survival, $\%$ & 28.6 & 38.1 & 0.120 & 42.9 & 35.8 & 0.510 \\
\hline HER 2 positive, $\%$ & 31.0 & 7.4 & 0.00001 & & $\mathrm{~N} / \mathrm{A}$ & \\
\hline HER 2 protein upregulation: IHC $2+$ and $3+, \%$ & 47.6 & 16.3 & 0.000008 & & N/A & \\
\hline
\end{tabular}

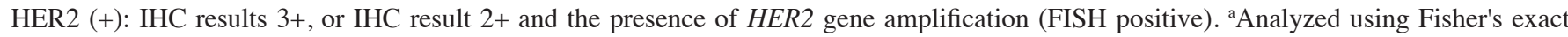
test. HER2, human epidermal growth factor receptor 2; CEP17, chromosome 17 centromere enumeration probe; CNI, copy number increase; IHC, immunohistochemistry; FISH, fluorescence in situ hybridization; N/A, not applicable; pT, depth of tumor invasion into the stomach wall; $\mathrm{pN}$, presence of nodal involvement.
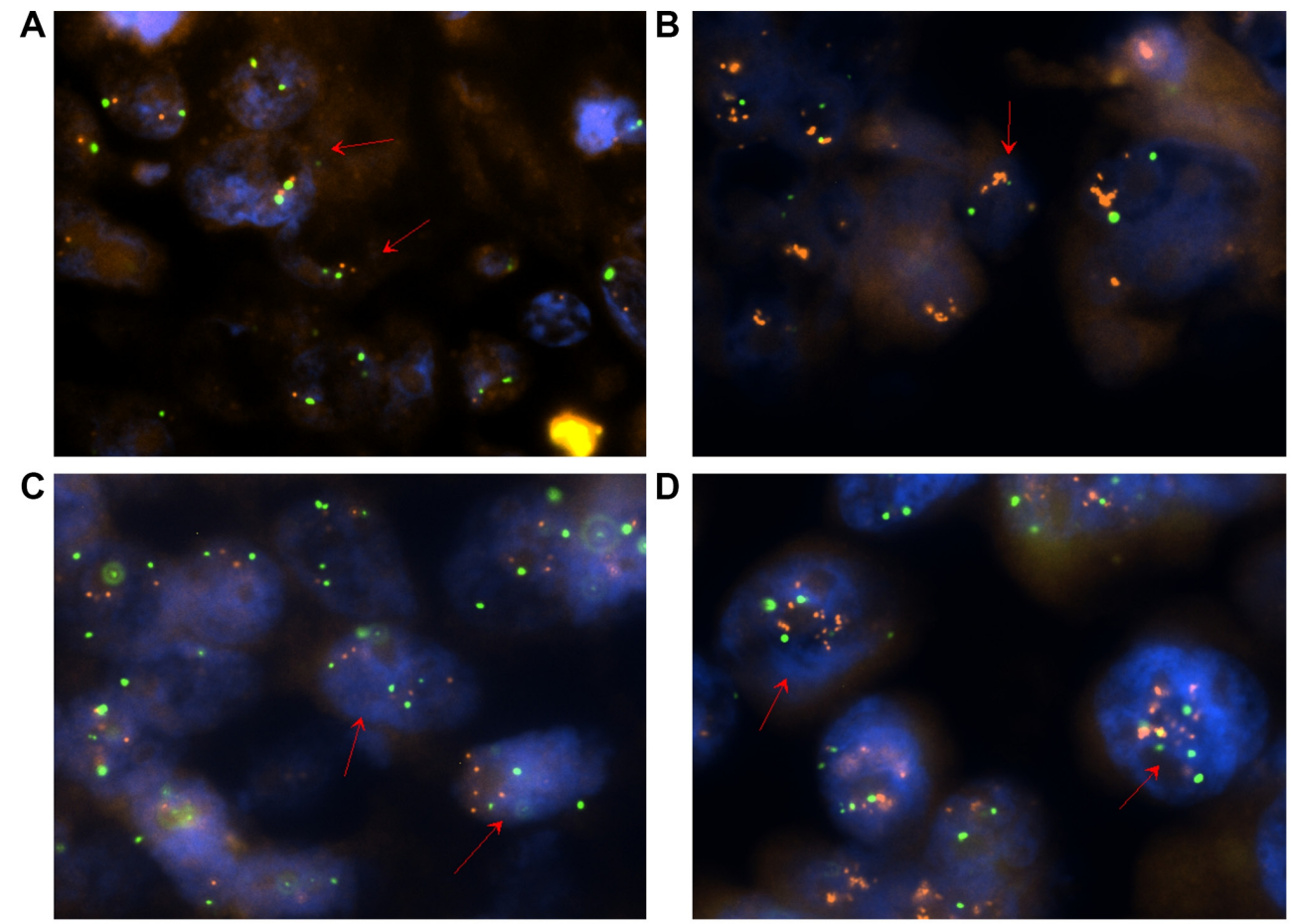

Figure 1. Dual-color FISH assays demonstrating HER2 gene copies (red) and CEP17 (green). (A) FISH-negative result (no amplification). Arrows indicate cells with two HER2 signals and two CEP17 signals. (B) FISH-positive result (amplification). Arrow indicates a cell with multiple HER2 signals and two CEP17 signals. (C) CEP17 CNI, FISH equivocal. Arrows indicate cells with five HER2 signals and three (right) and six (left) CEP17 signals. (D) CEP17 CNI and HER 2 gene amplification. Arrows indicate cells with multiple HER2 and CEP17 signals. FISH, fluorescence in situ hybridization; HER2, human epidermal growth factor receptor 2; CEP17, chromosome 17 centromere enumeration probe; CNI, copy number increase. 


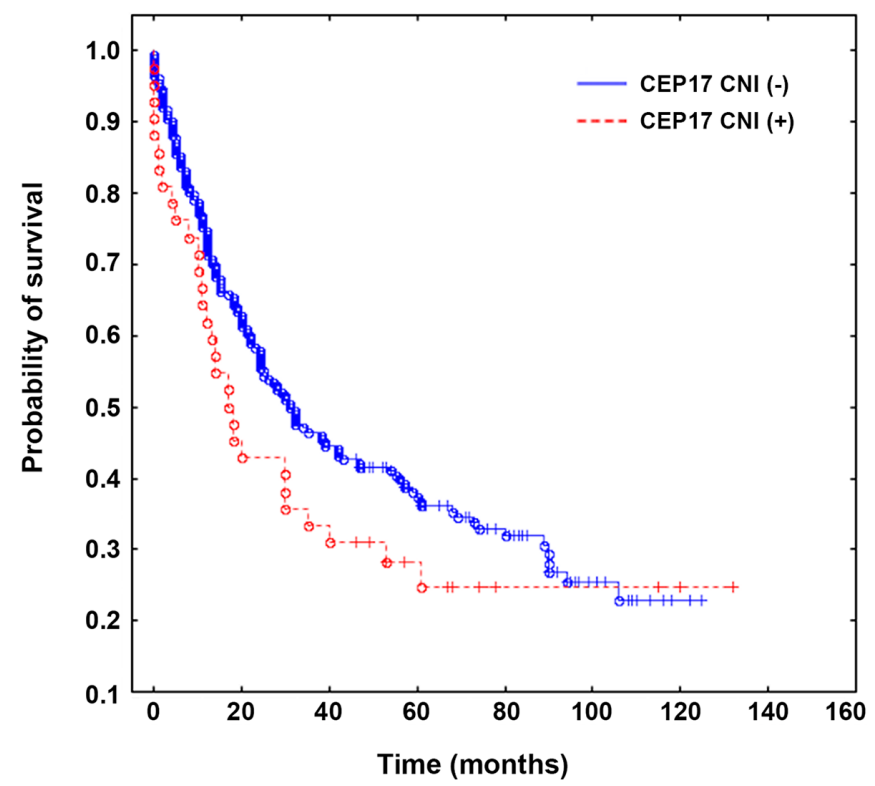

Figure 2. Comparison of overall survival rates of patients with the presence or absence of CEP17 CNI. P=0.17. CEP17, chromosome 17 centromere enumeration probe; $\mathrm{CNI}$, copy number increase.

Among all of the studied clinicopathological features, there was a significant difference between the CEP17-positive and -negative groups with regard to cardia involvement only $(\mathrm{P}=0.001$; Table I). However, there was also a strong association between CEP17 CNI and HER2 protein upregulation in the tumor cells $(\mathrm{P}<0.0001$; Table I).

Association between CEP17 CNI and patient survival. The Overall survival rate between the CEP17 CNI-positive and -negative groups was determined using the Kaplan-Meier method followed by the log-rank test; no significant difference was observed ( $\mathrm{P}=0.17$; Fig. 2 ). The two-year survival rate (also determined using the Kaplan-Meier method and log-rank test; Table I) tended to statistical significance in favor of CEP17 CNI-negative tumors $(\mathrm{P}=0.05)$.

HER2 positivity rate and the association between HER2 status and clinicopathological features. HER2 positivity was observed in $11.5 \%$ of cases and was equivocal in 4 cases (undetermined HER2 status). Among the 42 CEP17 CNI-positive cases, 13 were assessed as HER 2 positive, 3 as equivocal and 26 as negative. There were no significant differences between the HER2-positive and -negative groups regarding the range of stomach resection (rate of total gastrectomy, $89.3 \%$ vs. $87.3 \%$ ), the extent of lymphadenectomy (rate of D2-D1+, $17.9 \%$ vs. $25 \%$ ), the total number of lymph nodes resected (mean, 18.8 vs. 21.5; median 16 vs. 20), tumor location in the stomach, pTNM stage, pT, pN, the number of metastatic lymph nodes and the presence of distant metastases (Table I). HER2 status was significantly associated with intestinal type according to Lauren classification, and lack of mucinous component of the tumor. HER2 status was not associated with overall survival and survival rates. The relationships between HER2 status, CEP17 CNI and clinicopathological parameters are presented in Table I. The survival curves are presented in Figs. 2 and 3.

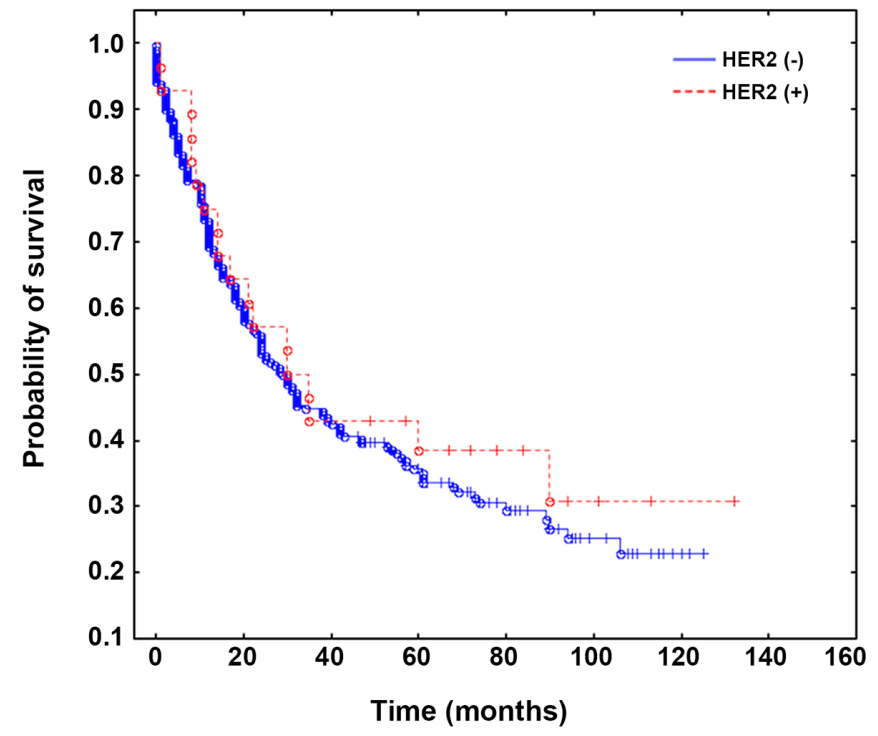

Figure 3. Comparison of overall survival rates of patients with HER2-negative and -positive status. $\mathrm{P}=0.49$. HER 2 , human epidermal growth factor receptor 2 .

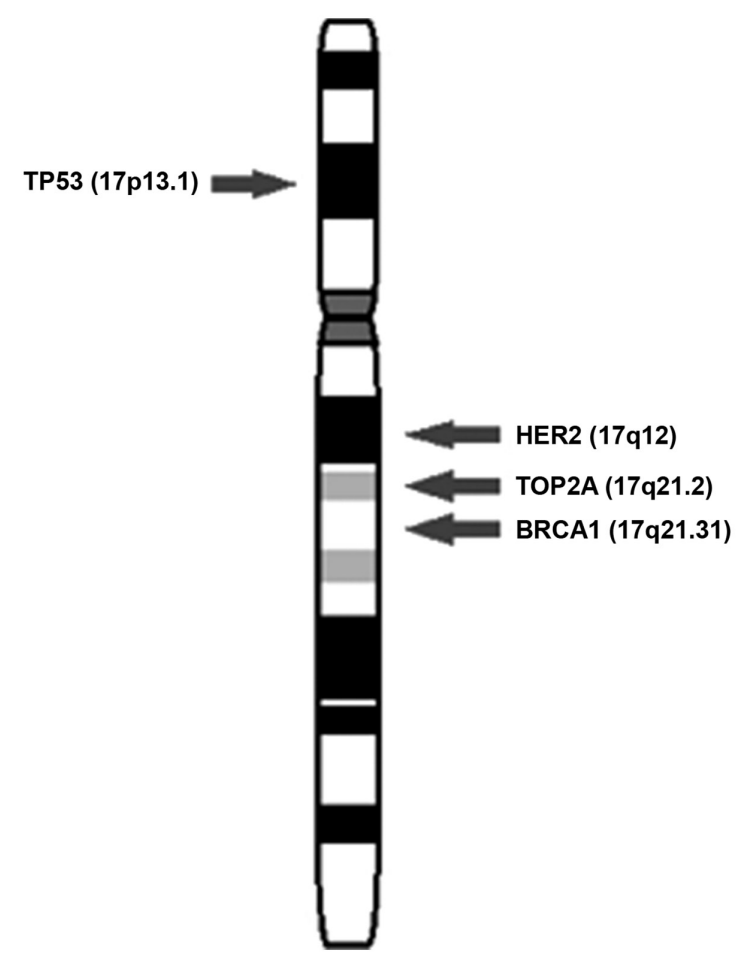

Figure 4. Chromosome 17 loci of HER2 and other genes associated with carcinogenesis. HER2, human epidermal growth factor receptor 2.

\section{Discussion}

Approval for the use of trastuzumab in patients with gastric cancer is as a result of HER2 upregulation, defined by an IHC score of $3+$, or $2+$ confirmed by a positive FISH result. These criteria have not changed over the years $(4,17)$. Positive HER2 status was observed in $20 \%$ of patients with gastric cancer (18), but ranged between 6.0 and $36.6 \%$ (19). The present study revealed HER 2 positivity in $11.5 \%$ of patients, as well as an association between positive HER2 status and 
the intestinal type, according to Lauren classification and lack of mucinous component of the tumor. These findings are consistent with those from a previous study (20), and other studies have also indicated a relationship between HER2 positivity and tumor location in the gastrointestinal junction $(8,21)$.

The HER2 oncogene is located on the long arm of the chromosome 17 , near the centromere (22), as shown in Fig. 4. FISH is performed with the use of dual probes, one for the HER2 gene and another for the centromere of chromosome 17 (23). Since the results of FISH are based on the ratio between the number of HER 2 gene and chromosome 17 centromere signals, a higher number of CEP17 signals translates to a lower HER2/CEP17 ratio. The issue of chromosome 17 copy number change contributing to a high percentage of inaccurate and equivocal results during HER2 status assessment, has already been raised in breast cancer (9).

Originally, CEP17 CNI was reported as chromosome 17 polysomy, but it is now recognized that true chromosome 17 polysomy, which is defined by the presence of extra copies of the whole chromosome, is an uncommon event in both breast and gastric cancers $(9,10,22-25)$. Indeed; the use of molecular tools such as multiple ligation probe amplification (MLPA) and array-comparative genomic hybridization have confirmed that in the vast majority of cases, elevated CEP17 signals are caused by an amplification of the centromeric region of the chromosome $(10,11,23,25-27)$. In this study, the HER2/CEP17 ratio was $<1$ in only a single case among 244 (data not shown), suggesting that the usually amplified centromeric region contains the HER2 gene locus on the long arm of the chromosome. Varga et al (28) performed FISH on 14 breast cancer specimens using multiple chromosome 17 probes, and demonstrated that CEP17 amplification almost always involves the HER2 locus. A strong relationship between CEP17 CNI and HER2 protein upregulation also suggests that an increased CEP17 signal is often associated with increased levels of HER2 gene expression in the cancer cell. The relationship between CEP17 CNI and IHC results was also found in other studies concerning both gastric cancer (19) and breast cancer (29). Therefore, the question of whether increased CEP17 copy signals should underrate FISH results arises.

In the present study, CEP17 CNI was found in $17.2 \%$ of cases and tended to be associated with poorer $1-(\mathrm{P}=0.12)$, 2- $(\mathrm{P}=0,05), 3-(\mathrm{P}=0.07), 4-(\mathrm{P}=0.1)$ and 5-year $(\mathrm{P}=0.12)$ survival rates. However, it must be stated that statistical analysis surrounding the impact of CEP17 CNI on survival did not reveal any significant differences. Apart from cardia involvement, CEP17 CNI was not significantly associated with any of the other investigated clinicopathological factors. The association between CEP17 CNI and clinicopathological features has not been widely studied. However, Onchi et al (30) revealed a relationship between CEP17 CNI and lymph node involvement.

The impact of CEP17 multiplication on adverse clinical outcomes or negative prognostic indicators has already been demonstrated in breast cancer $(11,12,31,32)$. Kim et al (11) revealed worse overall survival and disease free survival rates in breast cancer patients with non-amplified HER2 expression, but with CEP17 multiplication. Lee et al (12) found
CEP17 CNI to be an independent adverse prognostic factor in the HER2-negative tumors from 945 cases of invasive breast cancer. In this study, CEP17 CNI was also associated with multiple aggressive histological variables, including higher $\mathrm{T}$ stage, higher histologic grade, lymphovascular invasion, negative hormone receptor status, p53 upregulation and high Ki-67 proliferative index.

In addition to $H E R 2$, chromosome 17 contains other genes that participate in carcinogenic process, such as TOP $2 A$, DARPP32, BRCA1 and TP53 (25). The mechanisms facilitating the poor outcomes of CEP17 CNI-positive patients is not known, though the association between CEP17 CNI and HER2 upregulation begs us to question whether patients with CEP17 CNI-positive gastric cancer would benefit from anti-HER 2 therapy. If the answer is positive, in the study series of 244 patients, up to $29(11.9 \%$ ) polysomic, but HER2-negative $(n=26)$ or equivocal $(n=3)$ patients might have been denied eligible trastuzumab treatment.

In breast cancer, there is evidence that CEP17 CNI may determine the response to trastuzumab treatment in tumors with negative FISH results $(33,34)$. Hofmann et al (33) studied the response to trastuzumab first-line monotherapy in a group of 105 patients with HER2-positive metastatic breast cancer. A partial or complete response was observed in 19 of the $75(25.3 \%)$ patients with IHC $3+$ tumors, in 16 of 74 (21.6\%) patients with FISH-positive tumors, and in 6 of $26(23.1 \%)$ patients with CEP17 CNI. Notably, two of the six CNI-positive responders were FISH negative (HER2/CEP17 ratio <2,0). In a randomized study by Kaufman et al (34), trastuzumab was added to paclitaxel treatment in HER2-negative/CEP17 CNI-positive (CEP17 $\geq 2.2$ ) patients with metastatic breast cancer, and the response rate increased from 25 to $63 \%$. To further complicate matters, it is hypothesized that CEP17 CNI may serve different roles in the prediction of anti-HER2 treatment response for primary vs. metastatic breast cancer (35). Nevertheless, these data suggest that at least a proportion of patients with CEP17 CNI-positive breast cancer may potentially benefit from trastuzumab treatment, in spite of negative HER2 status.

In gastric cancer, the issue of CEP17 CNI in HER2 testing interpretation appears to be overlooked. Numerous studies have concluded that CEP17 CNI is infrequent and has limited impact on HER2 status evaluation (19). In the well-known ToGA trial (3) CEP17 CNI occurred in only $4.1 \%$ of the studied population. Similarly, Gomez-Martin et al (17) found only $2(3.0 \%)$ CEP17 CNI-positive and concurrent amplified cases among 66 patients fulfilling trastuzumab treatment criteria. The authors studied the impact of the level of HER2 gene amplification on the benefit to overall survival and the response to treatment with trastuzumab-based chemotherapy. Both CEP17 CNI-positive patients showed some degree of clinical benefit.

To the best of our knowledge, there are no studies concerning the response to trastuzumab in patients with HER2-negative, CEP17 CNI-positive gastric cancer. Such studies are highly anticipated to limit false-negative HER2 status assessment, and to optimize patient selection for HER2-targeted treatments.

The primary limitation of the present study was the lack of knowledge concerning systemic treatment of the studied patients. It is unclear whether the CEP17 CNI-positive and 
-negative groups were treated in the same manner. In the study period, neoadjuvant chemotherapy was not as widely adopted as it is now, and was received by only $8.2 \%$ of the patients. In the adjuvant settings, patients eligible for postoperative treatment primarily received chemoradiation in accordance with the MacDonald protocol (36). Due to the lack of standardized chemotherapy in recurrent and metastatic disease at this time, patients underwent multiple chemotherapeutic regimens according to the oncologist's judgement. In patients with metastatic gastric cancer, the reimbursement of Trastuzumab treatment costs by the Polish health care system began in on 1st March 2014, thus it is assumed that few, if any HER2-positive patients from this period in the study had received targeted anti-HER 2 therapy. There was also a lack of CEP17 CNI re-evaluation using novel molecular droplet digital PCR, MLPA or array techniques. On the other hand, the present study primarily focused on routinely used in situ hybridization techniques rather than technologies more frequently used in research.

In conclusion, the results of the present study indicate that CEP17 CNI is strongly associated with HER2 upregulation on tumor cells, thus it is recommended that the presence of CEP17 CNI be mentioned in routine histopathological reports. These findings may represent a critical issue in HER2 testing, complementing the clinical value of the HER2/CEP17 ratio for the prognosis and treatment of patients with gastric cancer. The impact of CEP17 CNI on HER2 upregulation is evident, and the eligibility for HER2-targeted agents in CEP17 CNI-positive patients requires further recognition.

\section{Acknowledgements}

The authors wish to profoundly thank Professor Marzena Anna Lewandowska from the Molecular Oncology and Genetics Department, IFM, Łukaszczyk Oncology Centre, Bydgoszcz for the FISH analyses and support in preparing the manuscript. Also, we graciously acknowledge Professor Janusz Jaśkiewicz and Professor Wojciech Biernat for their assistance in the study, as well as the Ministry of Digital Development for the disclosure of patient mortality dates.

\section{Funding}

The present study was financially supported by the Medical University of Gdańsk (grant no. ST 02/130/07/308).

\section{Availability of data and materials}

The datasets used and/or analyzed during the current study are available from the corresponding author on reasonable request.

\section{Authors' contributions}

MC conceived the presented idea, made major contributions to design of the study and wrote the manuscript. MSz analyzed the data, created the figures, and was involved in drafting the manuscript. JW made major contributions to data acquisition and interpretation. RP, RL and MSu performed all histopathological examinations (immunohistochemistry) and specimen preparation for FISH assessment. JZ and WJK made substantial contributions to the conception and design of the study, as well as the revision of the manuscript. All authors read and approved the final manuscript.

\section{Ethics approval and consent to participate}

The present study was approved by the Independent Ethics Committee of the Medical University of Gdańsk (NKBBN/427/2014), and the requirement for patient consent was waived by the committee.

\section{Patient consent for publication}

Not applicable.

\section{Competing interests}

The authors declare that they have no competing interests.

\section{References}

1. Bray F, Ferlay J, Soerjomataram I, Siegel RL, Torre LA and Jemal A: Global cancer statistics 2018: GLOBOCAN estimates of incidence and mortality worldwide for 36 cancers in 185 countries. CA Cancer J Clin 68: 394-424, 2018.

2. Jomrich G and Schoppmann SF: Targeted therapy in gastric cancer. Eur Surg 48: 278-284, 2016.

3. Bang YJ, Van Cutsem E, Feyereislova A, Chung HC, Shen L, Sawaki A, Lordick F, Ohtsu A, Omuro Y, Satoh T, et al: Trastuzumab in combination with chemotherapy versus chemotherapy alone for treatment of HER2-positive advanced gastric or gastro-oesophageal junction cancer (ToGA): A phase 3, open-label, randomised controlled trial. Lancet 376: 687-697, 2010.

4. Bartley AN, Washington MK, Colasacco C, Ventura CB, Ismaila N, Benson AB III, Carrato A, Gulley ML, Jain D, Kakar S, et al: HER2 testing and clinical decisions making in gastroesophageal adenocarcinoma: Guideline from the College of American Pahologists, American Society for Clinical Pathology, and the American Society of Clinical Oncology. J Clin Oncol 35: 446-464, 2017.

5. Boku N: HER2-positive gastric cancer. Gastric Cancer 17: 1-12, 2014.

6. Abrahao-Machado LF and Scapulatempo-Neto C: HER2 testing in gastric cancer: An update. World J Gastroenterol 22: 4619-4625, 2016

7. Rüschoff J, Dietel M, Barreton G, Arbogast S, Walch A, Monges G, Chenard M-P, Panault-LLorca F, Nagelmeier I, Schlake W, et al: HER2 diagnostics in gastric cancer-guideline validation and development of standardized immunohistochemical testing. Virchows Arch 457: 299-307, 2010.

8. Baretton G, Kreipe HH, Schirmacher P, Gaiser T, Hofheinz R, Berghäuser KH, Koch W, Künzel C, Morris S and Rüschoff J; Nicht-interventionelle Untersuchung (NIU) HER2 Study Group: HER2 testing in gastric cancer diagnosis: Insights on variables influencing HER2-positivity from a large, multicenter, observational study in Germany. Virchows Arch 474: 551-560, 2019.

9. Gunn S, Yeh IT, Lytvak I, Tirtorahardjo B, Dzidic N, Zadeh S, Kim J, McCaskill C, Lim L, Gorre M and Mohammed M: Clinical array-based karyotyping of breast cancer with equivocal HER2 status resolves gene copy number and reveals chromosome 17 complexity. BMC Cancer 10: 396-404, 2010.

10. Hanna WM, Rüschoff J, Bilous M, Coudry RA, Dowsett M, Osamura RY, Penault-Llorca F, van de Vijver M and Viale G: HER2 in situ hybridization in breast cancer: Clinical implications of polysomy 17 and genetic heterogeneity. Mod Pathol 27: 4-18, 2014

11. Kim A, Shin HC, Bae YK, Kim MK, Kang SH, Lee SJ and Lee EH: Multiplication of chromosome 17 centromere is associated with prognosis in patients with invasive breast cancer exhibiting normal HER2 and TOP2A status. J Breast Cancer 15: 24-33, 2012.

12. Lee K, Jang MH, Chung YR, Lee Y, Kang E, Kim S-W, Kim YJ, Kim JH, Kim IA and Park SY: Prognostic significance of centromere 17 copy number gain in breast cancer depends on breast cancer subtype. Hum Pathol 61: 111-120, 2017. 
13. Ciesielski M, Kruszewski WJ, Smiałek U, Walczak J, Szajewski M, Szefel J, Wydra J and Kawecki K: The HER2 gene and HER2 protein status and chromosome 17 polysomy in gastric cancer cells in own material. Appl Immunohistochem Mol Morphol 23: 113-117, 2015.

14. Edge S, Byrd DR, Compton CC, Fritz AG, Greene F and Trotti A (Eds.): American Joint Committee on Cancer, American Cancer Society, AJCC Cancer Staging Manual. 7th edition. Springer, New York, NY, 2010

15. Hofmann M, Stoss O, Shi D, Büttner R, van de Vijver M, Kim W, Ochiai A, Rüschoff $\mathbf{J}$ and Henkel T: Assessment of a HER2 scoring system for gastric cancer: Results from a validation study. Histopathology 52: 797-805, 2008.

16. Degiuli M, De Manzoni G, Di Leo A, D'Ugo D, Galasso E, Marrelli D, Petrioli R, Połom K, Roviello F, Santullo F and Morino M: Gastric cancer: Current status of lymph node dissection. World J Gastroenterol 22: 2875-2893, 2016.

17. Gomez-Martin C, Plaza JC, Pazo-Cid R, Salud A, Pons F, Fonseca P, Leon A, Alsina M, Visa L, Rivera F, et al: Level of HER 2 gene amplification predicts response and overall survival in HER2-positive advanced gastric cancer treated with trastuzumab. J Clin Oncol 31: 4445-4452, 2013.

18. McLean MH and El-Omar EM: Genetics of gastric cancer. Nat Rev Gastroenterol Hepatol 11: 664-674, 2014.

19. Yoshida H, Yamamoto N, Taniguchi H, Oda I, Katai H, Kushima R and Tsuda H: Comparison of HER2 status between surgically resected specimens and matched biopsy specimens of gastric intestinal-type adenocarcinoma. Virchows Arch 465: 145-154, 2014

20. Grabsch H, Sivakumar S, Gray S, Gabbert HE and Müller W. HER2 expression in gastric cancer: Rare, heterogeneous and of no prognostic value-conclusions from 924 cases of two independent series. Cell Oncol 32: 57-65, 2010.

21. Van Cutsem E, Bang YJ, Feng-yi F, Xu JM, Lee KW, Jiao SC, Chong JL, López-Sanchez RI, Price T, Gladkov O, et al: HER2 screening data from TOGA: Targeting HER2 in gastric and gastroesophageal junction cancer. Gastric Cancer 18: 476-484, 2015.

22. Wang T, Amemiya Y, Henry P, Seth A, Hanna W and Hsieh ET: Multiplex ligation-dependent probe amplification can clarify HER2 status in gastric cancer with 'polysomy 17'. J Cancer 6: 403-408, 2015

23. Yeh IT, Martin MA, Robetorye RS, Bolla AR, McCaskill C, Shah RK, Gorre ME, Mohammed MS and Gunn SR: Clinical validation of an array CGH test for HER 2 status in breast cancer reveals that polysomy 17 is a rare event. Mod Pathol 22: 1169-1175, 2009.

24. Sanguedolce $F$ and Bufo P: HER2 assessment by silver in situ hybridization: Where are we now? Expert Rev Mol Diagn 15 385-398, 2015

25. Koudelakova V, Trojanec R, Vrbkova J, Donevska S, Bouchalova K, Kolar Z, Varanasi L and Hajduch M: Frequency of chromosome 17 polysomy in relation to CEP17 copy number in a large breast cancer cohort. Genes Chromosomes Cancer 55: 409-417, 2016.
26. Marchiò C, Lambros MB, Gugliotta $\mathrm{P}$, Di Cantogno LV, Botta C, Pasini B, Tan DS, Mackay A, Fenwick K, Tamber N, et al: Does chromosome 17 centromere copy number predict polysomy in breast cancer? A fluorescence in situ hybridization and microarray-based CGH analysis. J Pathol 219: 16-24, 2009.

27. Moelans CB, de Weger RA and van Diest PJ: Absence of chromosome 17 polysomy in breast cancer: Analysis by CEP17 chromogenic in situ hybridization and multiplex ligation-dependent probe amplification. Breast Cancer Res Treat 120: 1-7, 2010.

28. Varga Z, Tubbs RR, Wang Z, Sun Y, Noske A, Kradolfer D, Bosshard G, Jochum W, Moch H and Öhlschlegel C: Co-amplification of the HER2 gene and chromosome 17 centromere: A potential diagnostic pitfall in HER2 testing in breast cancer. Breast Cancer Res Treat 132: 925-935, 2012.

29. Xu FP, Wang K, Xu J, Chen J, Zhang YF, Wu HM, Zhang MH, Long XX, Luo XL, Zhang KP, et al: Impact of repeat HER2 testing after initial equivocal HER2 FISH results using 2013 ASCO/CAP guidelines. Breast Cancer Res Treat 166: 757-764, 2017.

30. Onchi H, Hirose K, Yamaguchi A, Noriki S and Fukuda M: Prognostic value of numerical aberrations of chromosome 17 in differentiated gastric cancer: Evaluation by multivariate regression analysis. Oncol Rep 7: 1317-1322, 2000.

31. Krishnamurti U, Hammers JL, Atem FD, Storto PD and Silverman JF: Poor prognostic significance of unamplified chromosome 17 polysomy in invasive breast carcinoma. Mod Pathol 22: 1044-1048, 2009.

32. Orsaria M, Khelifa S,BuzaN, Kamath A and Hui P: Chromosome 17 polysomy: Correlation with histological parameters and HER2NEU gene amplification. J Clin Pathol 66: 1070-1075, 2013.

33. Hofmann M, Stoss O, Gaiser T, Kneitz H, Heinmöller P Gutjahr T, Kaufmann M, Henkel T and Rüschoff J: Central HER2 IHC and FISH analysis in a trastuzumab (Herceptin) phase II monotherapy study: Assessment of test sensitivity and impact of chromosome 17 polysomy. J Clin Pathol 61: 89-94, 2008.

34. Kaufman PA, Broadwater G, Lezon-Geyda K, Dressler LG, Berry D, Friedman P, Winer EP, Hudis C, Ellis MJ, Seidman AD and Harris LN: Correlation of HER2 and chromosome 17 (ch17) copy number with trastuzumab (T) efficacy in CALGB 9840, paclitaxel $(\mathrm{P})$ with or without $\mathrm{T}$ in HER2 ${ }^{+}$and HER2 metastatic breast cancer (MBC). J Clin Oncol 25: 1009, 2007.

35. Reinholz MM, Bruzek AK, Visscher DW, Lingle WL, Schroeder MJ, Perez EA and Jenkins RB: Breast cancer and aneusomy 17: Implications for carcinogenesis and therapeutic response. Lancet Oncol 10: 267-277, 2009.

36. Macdonald JS, Smalley SR, Benedetti J, Hundahl SA, Estes NC, Stemmermann GN, Haller DG, Ajani JA, Gunderson LL, Jessup JM and Martenson JA: Chemoradiotherapy after surgery compared with surgery alone for adenocarcinoma of the stomach or gastroesophageal junction. N Engl J Med 345: 725-730, 2001.

This work is licensed under a Creative Commons Attribution-NonCommercial-NoDerivatives 4.0 International (CC BY-NC-ND 4.0) License. 